\title{
Spatially Shaping Waves to Penetrate Deep inside a Forbidden Gap
}

\author{
Ravitej Uppu $\odot,{ }^{*}$ Manashee Adhikary $\odot$, Cornelis A. M. Harteveld®, and Willem L. Vos $\oplus^{\dagger}$ \\ Complex Photonic Systems (COPS), MESA+ Institute for Nanotechnology, University of Twente, \\ P.O. Box 217, 7500 AE Enschede, The Netherlands
}

(Received 21 July 2020; accepted 22 February 2021; published 27 April 2021)

\begin{abstract}
It is well known that waves with frequencies within the forbidden gap inside a crystal are transported only over a limited distance — the Bragg length—before being reflected by Bragg interference. Here, we demonstrate how to send waves much deeper into crystals in an exemplary study of light in twodimensional silicon photonic crystals. By spatially shaping the wave fronts, the internal energy densityprobed via the laterally scattered intensity-is enhanced at a tunable distance away from the front surface. The intensity is up to $100 \times$ enhanced compared to random wave fronts, and extends as far as $8 \times$ the Bragg length, which agrees with an extended mesoscopic model. We thus report a novel control knob for mesoscopic wave transport that pertains to any kind of waves.
\end{abstract}

DOI: 10.1103/PhysRevLett.126.177402

Completely controlling wave transport is a key challenge essential for a large variety of applications. For instance, classical transport of acoustic waves has enabled sensing, ultrasound imaging, and navigation [1,2]. In the quantum regime, control over electron and spin transport has brought major advances in the operation of nanoelectronic devices [3-5]. In photonics, control over light transport in the classical and quantum domains has led to rapid advances in science and technology such as solar cells, quantum light sources, optical memories, and microscale to nanoscale storage cavities [6-11].

An important tool in wave control is to exploit gap formation as a result of periodic and aperiodic symmetry. The long range periodic order leads to a band structure in the dispersion relations of the waves. Forbidden frequency ranges, stop gaps, emerge in the band structure as a result of interference between the incident and Bragg diffracted waves [12,13]. Bragg interference causes incident waves with a frequency in the stop gap to be exponentially attenuated, with a characteristic length scale called the Bragg length $L_{B}$, as is shown in Fig. 1. The existence of gaps has seen exciting applications such as control of spontaneous emission, efficient light harvesting devices, and biosensing $[14,15]$.

Real crystals feature unavoidable disorder resulting in broken symmetry due to, e.g., thermal motion and phonons in atomic crystals, quantum motion down to zero temperature, or unavoidable structural disorder in assembled

Published by the American Physical Society under the terms of the Creative Commons Attribution 4.0 International license. Further distribution of this work must maintain attribution to the author(s) and the published article's title, journal citation, and DOI. photonic or phononic structures [16-18]. The disorder gives rise to new channels for wave transport due to multiple scattering, which are typically uncontrolled and detrimental for applications. Every fabricated piece of crystal has a fixed microscopic configuration of disorder [19] and hence the disorder-induced channels do not offer new control. However, in disordered media without gaps spatially shaping the phases of incident waves sets interferences between the channels resulting in new control, termed wave front shaping [20-22].

In this Letter, we demonstrate the tunable control of wave transport in real photonic crystals. By spatially shaping the incident wave fronts as illustrated in Fig. 1, we steer the waves with frequencies within a gap to any desired location. The waves reach a remarkable depth of no less than $8 L_{B}$ with over $30 \times$ intensity enhancement. Remarkably, the shaped intensity at $5 L_{B}$ in the crystal is $10 \times$ higher than the incident intensity $I_{0}$ in the absence of wave shaping, instead of being Bragg attenuated in the crystal. We thus report a novel control knob for mesoscopic wave transport that pertains to any kind of waves, including

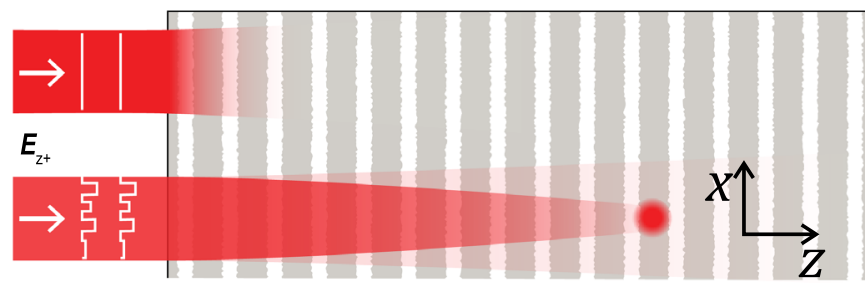

FIG. 1. Schematic of wave transport in a 2D photonic crystal consisting of pores with unavoidable disorder. At frequencies in the gap, light is quickly Bragg attenuated; by shaping the wave fronts, light is transported much deeper into the crystal. 
(a)

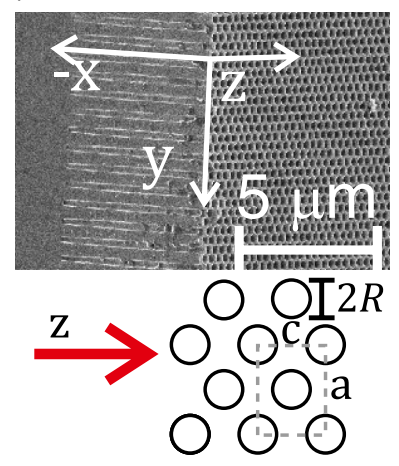

(b)

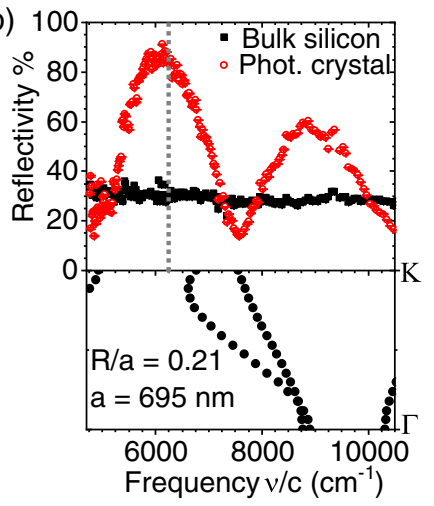

FIG. 2. (a) Left: scanning electron microscope image of a 2D photonic crystal. The lower half shows the $2 \mathrm{D}$ array of pores in the $y z$ plane, and the upper half shows the $x y$ cleavage plane with $6 \mu \mathrm{m}$ deep pores (scale bar: $5 \mu \mathrm{m}$ ). Right: $y z$ plane with the centered rectangular crystal with lattice parameters $a=695 \mathrm{~nm}$ and $c=a / \sqrt{2}$ with a pore diameter $2 R=290 \mathrm{~nm}$ (or $R / a=0.21$ ). Light is incident in the $z$ direction, corresponding to the $\Gamma K$ high-symmetry direction. (b) Reflectivity measured on the photonic crystal (open circles) for TE-polarized light, compared to unstructured silicon wafer (filled squares). The prominent photonic crystal reflectivity peaks match well with the stop gaps in the calculated band structure. The dashed line highlights the frequency near the gap center where many steering experiments were performed.

sound, spins, electrons, and electromagnetism, where similar techniques have been reported $[23,24]$.

Our results stand out from literature on wave front shaping and disordered media in the following aspects. (1) In photonic crystals, the energy density distribution within the crystal is completely fixed by the Bragg length. The introduction of wave front shaping radically modifies this distribution and increases the depth to which light can be steered, thereby increasing the functional volume [25]. Modifying the energy density distribution is also new compared to disordered media that lack frequency gaps. (2) In disordered media, the intensity inside the medium after wave front shaping is everywhere less than at the front surface before wave front shaping. Here, we strikingly observe that upon wave front shaping to a location inside the crystal, the intensity within the photonic crystal is even greater than at the front surface measured for an uncontrolled wave front. (3) We identify that Bragg interference - typical of periodic media including quasicrystals - take on a novel role in mesoscopic physics, that of reducing the number of channels for wave transport.

We realize this physics with light waves in two-dimensional (2D) photonic crystals with large periodic arrays of pores etched in a silicon wafer [27,28], see Fig. 2(a). The pores are made using deep reactive ion etching through an etch mask. The lateral $y z$ extent of the $2 \mathrm{D}$ crystal is $10 \times$ $10 \mathrm{~mm}^{2}$ at the center of the wafer, much larger than the expected Bragg and scattering length scales. The fabricated

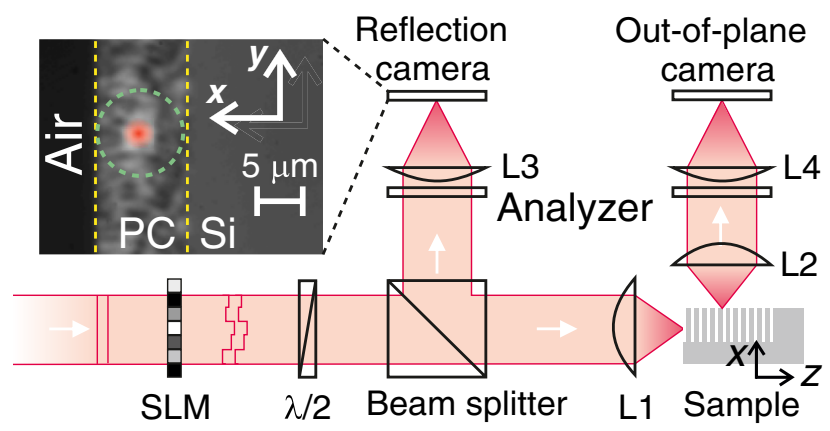

FIG. 3. Linearly polarized light from a tunable source is directed via a spatial light modulator (SLM) onto the sample through microscope objective $M 1$. The incident polarization is tuned using the half-wave plate $(\lambda / 2)$. Light reflected by the crystal is imaged onto an infrared camera using the same objective and lens $L 1(f=500 \mathrm{~mm})$. A long working distance objective (M2) images the lateral scattered light onto an infrared camera using lens $L 2(f=500 \mathrm{~mm})$. Broadband linear polarizers are used to analyze the reflected and the lateral scattered light. The inset shows the image taken on the camera in reflection. The bright spot in the center (highlighted in red) is the focused coherent light with a uniform phase pattern on the SLM.

pores are about $6 \mu \mathrm{m}$ deep, sufficient for the focus to easily fit within the crystal. The wafers are cleaved in the $x y$ plane to expose the 2D photonic crystal to the incident light along the $\Gamma K$ high-symmetry direction [28].

We developed a versatile experimental setup to perform optical wave front shaping on silicon nanostructures, which operates in the near-infrared spectral range $(\lambda>1100 \mathrm{~nm})$ where silicon absorption is avoided. The setup consists of three main components: (1) a broadband tunable coherent source, (2) a broadband wave front shaper, and (3) a twinarm imaging of reflected and lateral (yz plane) scattered signal from the photonic crystal. The broadband tunable coherent source was realized by spectrally filtering the emission from a supercontinuum source using a monochromator. A long pass filter (cut-on wavelength: $850 \mathrm{~nm}$ ) rejects the second-order diffraction of shorter wavelengths. The optical frequencies range from 4700 to $11000 \mathrm{~cm}^{-1}$ (or wavelengths $900<\lambda<2120 \mathrm{~nm}$ ) with a bandwidth of $0.6 \pm 0.1 \mathrm{~nm}$ and a tuning resolution of $0.2 \mathrm{~nm}$. Since the spectral bandwidth of the source corresponds to a coherence length of several hundred micrometers, much longer than the propagation length measured within the crystal, the pulsed nature of the source does not affect the measurements. The filtered light is collimated and expanded to a beam diameter of $7.5 \mathrm{~mm}$.

The wave front of the collimated beam is then programmed employing a phase-only reflective spatial light modulator SLM (Meadowlark optics), see Fig. 3. The wave front-programmed light is imaged to the back focal plane of the large numerical aperture $(\mathrm{NA}=0.85)$ infrared apochromatic objective lens $L 1$. The light reflected from the crystal is collected by the same objective. The lateral 
scattered light from the $y z$ plane of the crystal is collected using another apochromatic objective $L 2(\mathrm{NA}=0.42)$. Light collected by either $L 1$ or $L 2$ can be imaged to an InGaAs camera for aligning the incident beam. The inset in Fig. 3 shows the image captured in reflection, where the air, photonic crystal, and the unstructured silicon regions can be clearly distinguished. The bright spot in the center of the photonic crystal is the focused laser beam when a constant phase $(\phi=0)$ was programmed on the SLM. Given the large depth $(>6 \mu \mathrm{m})$ of the nanopores forming the photonic crystal, several diffraction-limited spots fit within any incident area on the crystal. Therefore, in contrast to earlier wave front shaping on 2D disordered waveguides that utilized one-dimensional wave front shaping [29-31], we illuminate a circular area $(d=8 \mu \mathrm{m}$, see Fig. 3$)$.

Reflectivity from the 2D crystal was measured following the procedures described in Ref. [32]. TE-polarized reflectivity spectra shown in Fig. 2(b) reveal two prominent peaks that correspond to two stop gaps in the calculated band structures. We estimate the Bragg lengths for both gaps from the photonic strength $S$ using the relation $L_{B}=$ $\lambda /(\pi S)$ [33], where $S \equiv \Delta \nu / \nu$ [34]. In the two stop bands at $\nu / c=6250 \mathrm{~cm}^{-1}$ and $9000 \mathrm{~cm}^{-1}$, respectively, the photonic strength is $S=0.25$ and 0.19 , respectively, corresponding to Bragg lengths $L_{B}=2.0 \mu \mathrm{m}$ that are nearly the same for both stop gaps [35].

To demonstrate controlled transport of waves, we tune the frequency of the incident light to the center of the first stop gap at $\nu / c=6250 \mathrm{~cm}^{-1}(\lambda=1600 \mathrm{~nm})$. The SLM was initialized with a phase pattern of 340 segments (each grouped from $32 \times 32$ pixels) within the beam diameter, each assigned a random phase within $[0,2 \pi)$. The size of the segment was chosen to limit the illuminated area on the $x y$ plane of the sample to be confined within the photonic crystal. The image of the lateral scattered light collected using $L 2$ is shown in Fig. 4(a), which shows a rapid decay of light into the crystal. The input edge of the crystal is clearly discernible as the bright speckles (also marked by the dashed line).

We target a spot at a depth of $10 \mu \mathrm{m}-$ or $5 L_{B}$-in the crystal and sequentially change the phase of each segment on the SLM to maximize the intensity at the chosen spot. Figure 4(b) shows the image of the lateral scattered light at the end of the iteration over all the segments. A distinct and bright focus is clearly observed at the targeted location, thereby demonstrating the first ever steering of waves inside a crystal, far beyond the Bragg length. Figure 4(c) shows the intensity around the targeted spot before and after steering the light, which shows $\approx 100 \times$ higher intensity with controlled transport.

In the literature, it is common to characterize the quality of wave front shaping by an enhancement $E_{W}$ that gauges the intensity increase at the target position [36,37]. The enhancement is commonly defined as $E_{W} \equiv I_{\text {opt }} /\left\langle I_{r}\right\rangle$, where $I_{\text {opt }}$ is the optimized intensity in the target spot
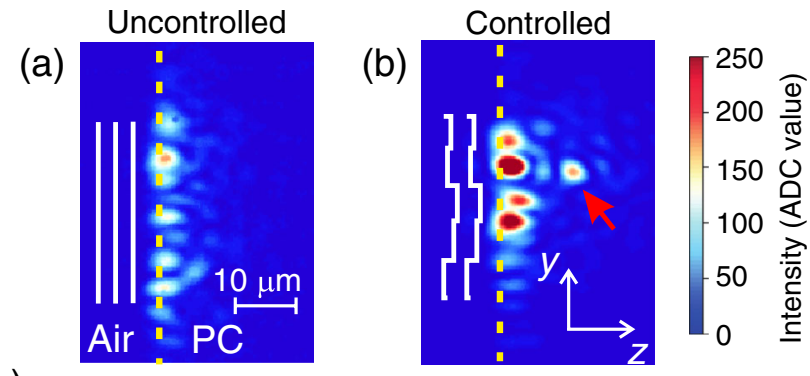

(c)

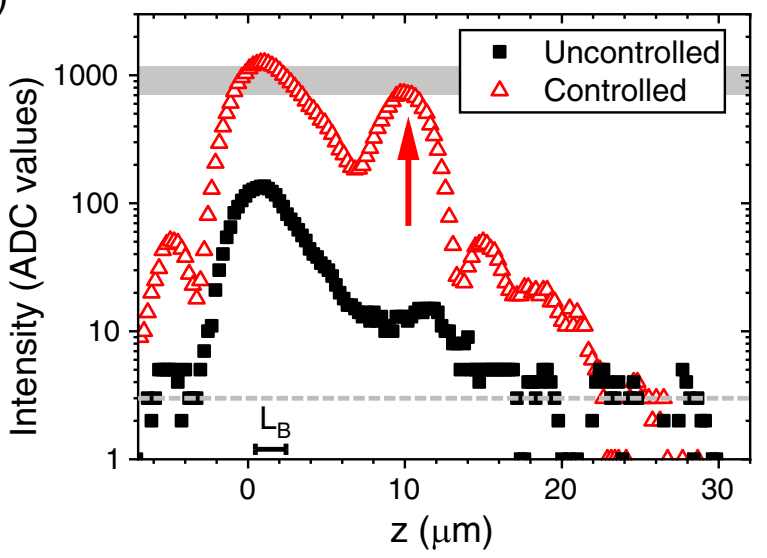

FIG. 4. Camera images of the lateral scattered light $(\nu / c=$ $6250 \mathrm{~cm}^{-1}$ ) with (a) an uncontrolled and (b) the controlled wave front. The red arrow points to the target location for steering the light, which exhibits as a bright spot. (c) The integrated intensity in a 5 pixel high ( $y z$ plane) strip around the target location shows a clear increase in the intensity at the target depth of $10 \mu \mathrm{m}$. The dashed gray line indicates the detector dark counts. The gray band is the intensity expected at $z=10 \mu \mathrm{m}$ upon wave front shaping as predicted by our model.

and $\left\langle I_{r}\right\rangle$ is the intensity at the same spot that was ensemble averaged over 100 random incident wave front patterns. The intensity of the lateral scattered light is proportional to the energy density of the light that has scattered to a depth $z$ in the crystal. The enhancement at the target location inside the crystal is substantial, namely $E_{W}=65 \pm 5$. Remarkably, in Fig. 4 we observe that the intensity at the target spot (depth $\left.z_{\text {opt }}=5 L_{B}\right)$ after steering is even $10 \times$ greater than the intensity at the front surface $(z=0)$ before steering (that is, light incident with an unoptimized wave front), in contrast to traditional Bragg attenuation of waves inside any periodic medium. The enhancements observed here are a key highlight of the steering of waves, which utilizes multiple scattering in the crystal arising from the unavoidable deviations from perfect periodicity [38]. The multiple scattering of waves gives rise to new channels for wave transport within the crystal even inside the stop gap. We selectively enhance the coupling of energy to these new transport channels by shaping the phases of the incident waves with the feedback defined by the energy density deep inside the crystal. This selective coupling results in propagation of waves deeper into the crystal. The remarkable increase in the total energy 


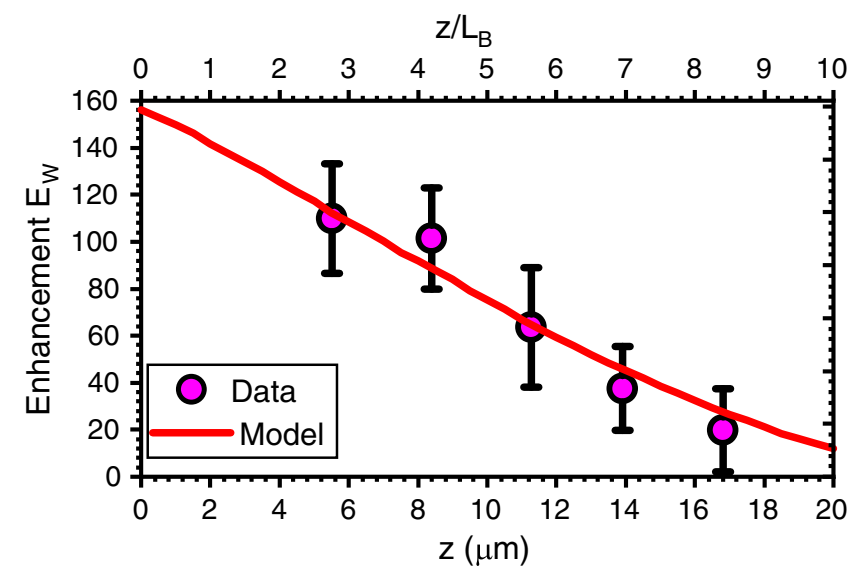

FIG. 5. Measured wave front shaping enhancement $E_{W}$ versus penetration depth $z$ into the crystal at a frequency at the center of the stop gap $\nu / c=6250 \mathrm{~cm}^{-1}$. The calculated enhancement using a model that accounts for the Bragg interference and the random multiple scattering agrees well with the measurements.

density while optimizing for a target location is in contrast to all prior wave front shaping experiments on random media, which highlights the new physics of light transport unveiled here.

We explored the maximum achievable depth inside the photonic crystal at which the intensity could be enhanced. Figure 5 shows the depth-dependent intensity enhancement $E_{W}$ at the center of the stop gap (at $\nu / c=6250 \mathrm{~cm}^{-1}$ ). The error bars represent standard deviations of $E_{W}$ measured at eight spatial locations along $y$ at the same depth $z$. An enhancement $E_{W}$ in excess of 80 was achieved at depths up to $10 \mu \mathrm{m}$, corresponding to $5 L_{B}$. At a depth of more than $8 L_{B}$, the measured enhancement is $E_{W}=20$.

To interpret the intensity enhancement deep inside the crystal, we propose a new model that extends mesoscopic physics typical of disordered media [22,39] to periodic crystals. The disorder-assisted multiple scattering of light inside the crystal is described using a transmission matrix $T$ over the $N$ transport channels. The transmission matrix $T$ can be decomposed as $T=U D V^{\dagger}$, where $U$ and $V$ are unitary matrices of size $N \times N$ and $D$ is a diagonal matrix with values $\tau$ drawn from the Dorokhov-Mello-PereyraKumar (DMPK) distribution $P_{\mathrm{DMPK}}(\tau)=\langle\tau\rangle /(2 \tau \sqrt{1-\tau})$, where $\langle\tau\rangle$ is the mean transmission [39-42]. The reflectivity measurements of the photonic crystal, cf. Fig. 2(b), were used to extract the fraction of light transmitted into the crystal. We neglect the material absorption of waves within the crystal given the near-zero absorption coefficient of silicon in the telecom wavelength range. The wave front shaping enhancement $E_{W}$ is proportional to the largest eigenvalue of the matrix $T$, with the proportionality constant set by the wave front shaping method [21]. Typically, the local energy density within a disordered slab decreases approximately linearly with depth inside the slab. In contrast, we observe from Fig. 4(c) that the local energy density decays exponentially with depth inside the crystal. We interpret this exponential attenuation to arise from the presence of Bragg interference at frequencies within the stop gap and the surface losses, with the Bragg interference being the dominant factor. The Bragg interference results in a reduction of the number of transport channels with increasing depth inside the crystal, thereby allowing us to extend mesoscopic transport models developed for disordered media in the presence of absorption [43,44] to periodic systems.

Mathematically, the reduction in the number of transport channels is modeled as a truncation of the transmission matrix $T$, i.e., a reduced matrix size $M<N$. The reduced number of channels $M$ with increasing depth in the crystal is modeled as $M(z)=N(1-\alpha z)$, where $\alpha$ is an amplitude attenuation constant [45]. The depth-dependent enhancement $E_{W}(z)$ is the maximum eigenvalue of the truncated transmission matrix. At a given $\alpha$ and $N$, we employ a numerical algorithm to generate an ensemble of random transmission matrices and compute the depth-dependent enhancement $E_{W}$. The fit to the measured enhancement $E_{W}$ is shown in Fig. 5 with $\alpha=(0.05 \pm 0.002) \mu \mathrm{m}^{-1}$ and $N=300$, and is seen to agree very well with the data. To put our model in perspective, the number of control parameters (segments) on the SLM is $N_{\exp }=340$, which agrees remarkably well with the number of channels in the model. The deviation could arise from the nonuniform intensity incident at each segment of the SLM due to the Gaussian profile of the incident beam. From an independent measurement of the attenuation within the crystal, we extract the intensity extinction length $\ell_{\text {ext }}=6.5 \pm 0.2 \mu \mathrm{m}$ (see Supplemental Material) arising from the multiple scattering of waves. The intensity attenuation length and the amplitude attenuation constant are related as $\ell_{\text {ext }}=1 / \sqrt{\alpha}$. From the fit to $E_{W}$ in Fig. 5, we estimate $\ell_{\text {ext }}=4.9 \pm 0.5 \mu \mathrm{m}$, which is smaller than the measured value. The underestimation of $\ell_{\text {ext }}$ is expected as the model effectively accounts for the attenuation due to the Bragg interference and multiple scattering of waves within the crystal, while the measured value is primarily due to the multiple scattering of waves. We note that $\ell_{\text {ext }}$ extracted from the model fits is greater than the Bragg length, as the disordered-induced transport within the crystal is polarization averaged, whereas the Bragg length pertains strictly to TE-polarized light.

In summary, we have demonstrated controllable enhancement of wave propagation at much greater depths than a Bragg length, even at frequencies within a band gap [46]. We take advantage of transport channels that are introduced in the crystal by unavoidable disorder, and address these by spatially shaping the wave fronts. The large depth to which waves are enhanced, even within a gap, broadens the range of applications feasible with photonic band gap crystals, both $2 \mathrm{D}$ and $3 \mathrm{D}$. From the outset, photonic band gaps have been pursued for their 
radical control over spontaneous emission $[47,48]$, lasing [8], shielding of vacuum noise for qubits [49], and for ultimate $3 \mathrm{D}$ waveguiding [50,51]. Based on our observations and modeling, we project that studies with embedded emitters could resolve the density of states around the gap of the real crystal [52,53]. Furthermore, light can be reconfigurably steered to resonant and functional features, including Anderson localized states [54] or "Cartesian" light states [55], that are otherwise hidden inside a complete 2D or 3D photonic band gap.

Recently, combinations of gaps and tailored disorder are enjoying fast-growing attention [56], notably in phononic and photonic quasicrystals [57], and hyperuniform [58,59] and biomimetic structures [60]. It is intriguing to speculate whether our crystals may reveal extremal transmission near a Dirac point and pseudodiffuse behavior as predicted by Sepkhanov et al. on 2D crystals [61]. Therefore our results open new avenues to increased wave control in many different classes of metamaterials $[62,63]$.

We thank Diana Grishina, Ad Lagendijk, Willemijn Luiten, Femi Ojambati, and Allard Mosk (Utrecht) for helpful comments and experimental help. We acknowledge support from NWO-FOM program Nr. 138 "Stirring of Light!," NWO-TTW Perspectief program P15-36 "Free form scattering optics" (FFSO), NWO ENW-GROOT program "Self-assembled icosahedral photonic quasicrystals with a band gap for visible light" OCENW.GROOT.2019.071, the MESA + Institute section Applied Nanophotonics (ANP), and the Center for Hybrid Quantum Networks (Hy-Q; DNRF-139), Niels Bohr Institute, University of Copenhagen, led by Peter Lodahl for supporting RU during a postdoctoral stay.

*Corresponding author.

ravitej-uppu@uiowa.edu

Present address: Department of Physics and Astronomy, The University of Iowa, Iowa City, Iowa 52242, USA. †.1.vos@utwente.nl

[1] M. Fink, D. Cassereau, A. Derode, C. Prada, P. Roux, M. Tanter, J.-L. Thomas, and F. Wu, Rep. Prog. Phys. 63, 1933 (2000).

[2] S. A. Cummer, J. Christensen, and A. Alù, Nat. Rev. Mater. 1, 16001 (2016).

[3] S. Neusser and D. Grundler, Adv. Mater. 21, 2927 (2009).

[4] K. Wagner, A. Kákay, K. Schultheiss, A. Henschke, T. Sebastian, and H. Schultheiss, Nat. Nanotechnol. 11, 432 (2016).

[5] K. Klyukin, L. L. Tao, E. Y. Tsymbal, and V. Alexandrov, Phys. Rev. Lett. 121, 056601 (2018).

[6] M. Aspelmeyer, T. J. Kippenberg, and F. Marquardt, Rev. Mod. Phys. 86, 1391 (2014).

[7] W. Li and S. Fan, Opt. Express 26, 15995 (2018).

[8] A. Tandaechanurat, S. Ishida, D. Guimard, M. Nomura, S. Iwamoto, and Y. Arakawa, Nat. Photonics 5, 91 (2011).
[9] J. Wang, F. Sciarrino, A. Laing, and M. G. Thompson, Nat. Photonics 14, 273 (2020).

[10] E. Kuramochi, K. Nozaki, A. Shinya, K. Takeda, T. Sato, S. Matsuo, H. Taniyama, H. Sumikura, and M. Notomi, Nat. Photonics 8, 474 (2014).

[11] A. F. Koenderink, A. Alù, and A. Polman, Science 348, 516 (2015).

[12] N. W. Ashcroft and N. D. Mermin, Solid State Physics (Holt, Rinehart, and Winston, New York, 1976).

[13] J. D. Joannopoulos, S. G. Johnson, J. N. Winn, and R. D. Meade, Photonic Crystals: Molding the Flow of Light (Princeton University Press, Princeton, NJ, 2011).

[14] R. B. Wehrspohn and J. Üpping, J. Opt. 14, 024003 (2012).

[15] H. Inan, M. Poyraz, F. Inci, M. A. Lifson, M. Baday, B. T. Cunningham, and U. Demirci, Chem. Soc. Rev. 46, 366 (2017).

[16] A. F. Koenderink and W. L. Vos, Phys. Rev. Lett. 91, 213902 (2003).

[17] E. Akkermans and G. Montambaux, Mesoscopic Physics of Electrons and Photons (Cambridge University Press, Cambridge, England, 2007).

[18] D. Wiersma, Nat. Photonics 7, 188 (2013).

[19] D. A. Grishina, C. A. M. Harteveld, A. Pacureanu, D. Devashish, A. Lagendijk, P. Cloetens, and W. L. Vos, ACS Nano 13, 13932 (2019).

[20] A. P. Mosk, A. Lagendijk, G. Lerosey, and M. Fink, Nat. Photonics 6, 283 (2012).

[21] I. M. Vellekoop, Opt. Express 23, 12189 (2015).

[22] S. Rotter and S. Gigan, Rev. Mod. Phys. 89, 015005 (2017).

[23] J. Harris, V. Grillo, E. Mafakheri, G. C. Gazzadi, S. Frabboni, R. W. Boyd, and E. Karimi, Nat. Phys. 11, 629 (2015).

[24] Y. Xie, W. Wang, H. Chen, A. Konneker, B.-I. Popa, and S. A. Cummer, Nat. Commun. 5, 5553 (2014).

[25] The functional volume is the total useful volume of all functional light-matter interfaces (e.g., cavities) embedded within the crystal. This volume is ideally as large as possible, and should not be confused with the mode volume of a cavity that is typically as small as possible, see, e.g., [26].

[26] J.-M. Gérard, Solid-state cavity-quantum electrodynamics with self-assembled quantum dots, in Single Quantum Dots, Topics in Applied Physics, Vol. 90, edited by P. Michler (Springer, New York, 2003), pp. 269-315.

[27] L. A. Woldering, R. W. Tjerkstra, H. V. Jansen, I. D. Setija, and W. L. Vos, Nanotechnology 19, 145304 (2008).

[28] S. R. Huisman, R. V. Nair, A. Hartsuiker, L. A. Woldering, A. P. Mosk, and W. L. Vos, Phys. Rev. Lett. 108, 083901 (2012).

[29] R. Sarma, A. G. Yamilov, S. Petrenko, Y. Bromberg, and H. Cao, Phys. Rev. Lett. 117, 086803 (2016).

[30] R. Sarma, A. Yamilov, and H. Cao, Appl. Phys. Lett. 110, 021103 (2017).

[31] P. Fang, C. Tian, L. Zhao, Y. P. Bliokh, V. Freilikher, and F. Nori, Phys. Rev. B 99, 094202 (2019).

[32] M. Adhikary, R. Uppu, C. A. Harteveld, D. A. Grishina, and W. L. Vos, Opt. Express 28, 2683 (2020).

[33] W. L. Vos, A. Lagendijk, and A. P. Mosk, Light propagation and emission in complex photonic media, in Light Localisation and Lasing: Random and Quasi-random Photonic Structures, edited by M. Ghulinyan and L. Pavesi (Cambridge 
University Press, Cambridge, England, 2014), pp. 1-12, https://doi.org/10.1017/CBO9781139839501.002.

[34] W. L. Vos, R. Sprik, A. van Blaaderen, A. Imhof, A. Lagendijk, and G. H. Wegdam, Phys. Rev. B 53, 16231 (1996).

[35] See Supplemental Material at http://link.aps.org/ supplemental/10.1103/PhysRevLett.126.177402 for frequency-dependent Bragg length and extinction length measurements, which includes Refs. [27,28].

[36] I. M. Vellekoop and A. P. Mosk, Phys. Rev. Lett. 101, 120601 (2008).

[37] S. M. Popoff, A. Goetschy, S. F. Liew, A. D. Stone, and H. Cao, Phys. Rev. Lett. 112, 133903 (2014).

[38] A. F. Koenderink, A. Lagendijk, and W. L. Vos, Phys. Rev. B 72, 153102 (2005).

[39] F. Evers and A. D. Mirlin, Rev. Mod. Phys. 80, 1355 (2008).

[40] O. N. Dorokhov, Solid State Commun. 51, 381 (1984).

[41] P. Mello, P. Pereyra, and N. Kumar, Ann. Phys. (N.Y.) 181, 290 (1988).

[42] D. Akbulut, T. Strudley, J. Bertolotti, E. P. A. M. Bakkers, A. Lagendijk, O. L. Muskens, W. L. Vos, and A. P. Mosk, Phys. Rev. A 94, 043817 (2016).

[43] A. Goetschy and A. D. Stone, Phys. Rev. Lett. 111, 063901 (2013).

[44] C. W. Hsu, A. Goetschy, Y. Bromberg, A. D. Stone, and H. Cao, Phys. Rev. Lett. 115, 223901 (2015).

[45] J. B. Pendry, A. MacKinnon, and A. B. Prêtre, Physica (Amsterdam) 168A, 400 (1990).

[46] We expect qualitatively similar results for light incident along the orthogonal crystal direction as the crystal also supports a gap along the $\Gamma M$ direction. Since our crystal thus has a 2D band gap, the results hold for a 2D band gap, and likely also for a full 3D band gap.

[47] P. Lodahl, A. F. Van Driel, I. S. Nikolaev, A. Irman, K. Overgaag, D. Vanmaekelbergh, and W. L. Vos, Nature (London) 430, 654 (2004).

[48] M. Fujita, S. Takahashi, Y. Tanaka, T. Asano, and S. Noda, Science 308, 1296 (2005).
[49] A. A. Clerk, M. H. Devoret, S. M. Girvin, F. Marquardt, and R. J. Schoelkopf, Rev. Mod. Phys. 82, 1155 (2010).

[50] S. A. Rinne, F. García-Santamaría, and P. V. Braun, Nat. Photonics 2, 52 (2008).

[51] K. Ishizaki, M. Koumura, K. Suzuki, K. Gondaira, and S. Noda, Nat. Photonics 7, 133 (2013).

[52] N. Vats, S. John, and K. Busch, Phys. Rev. A 65, 043808 (2002).

[53] C. P. Mavidis, A. C. Tasolamprou, S. B. Hasan, T. Koschny, E. N. Economou, M. Kafesaki, C. M. Soukoulis, and W. L. Vos, Phys. Rev. B 101, 235309 (2020).

[54] P. W. Anderson, Phys. Rev. 109, 1492 (1958).

[55] S. A. Hack, J. J. W. van der Vegt, and W. L. Vos, Phys. Rev. B 99, 115308 (2019).

[56] S. F. Liew, J.-K. Yang, H. Noh, C. F. Schreck, E. R. Dufresne, C.S. O'Hern, and H. Cao, Phys. Rev. A 84, 063818 (2011).

[57] A. Ledermann, L. Cademartiri, M. Hermatschweiler, C. Toninelli, G. A. Ozin, D. S. Wiersma, M. Wegener, and G. Von Freymann, Nat. Mater. 5, 942 (2006).

[58] W. Man, M. Florescu, E. P. Williamson, Y. He, S. R. Hashemizad, B. Y. Leung, D. R. Liner, S. Torquato, P. M. Chaikin, and P. J. Steinhardt, Proc. Natl. Acad. Sci. U.S.A. 110, 15886 (2013).

[59] N. Muller, J. Haberko, C. Marichy, and F. Scheffold, Optica 4, 361 (2017).

[60] S. Vignolini and N. Bruns, Adv. Mater. 30, 1801687 (2018).

[61] R. A. Sepkhanov, Y. B. Bazaliy, and C. W. J. Beenakker, Phys. Rev. A 75, 063813 (2007).

[62] S. Yves, R. Fleury, T. Berthelot, M. Fink, F. Lemoult, and G. Lerosey, Nat. Commun. 8, 16023 (2017).

[63] T. Ozawa, H. M. Price, A. Amo, N. Goldman, M. Hafezi, L. Lu, M. C. Rechtsman, D. Schuster, J. Simon, O. Zilberberg, and I. Carusotto, Rev. Mod. Phys. 91, 015006 (2019). 